\title{
Quality of food consumption and development of eating disorders among people living with HIV
}

\author{
Alongkote Singhato ${ }^{1}$, Uraiporn Booranasuksakul ${ }^{1}$, Narisa Rueangsri ${ }^{1}$, Gene Charoonruk ${ }^{2}$ \\ ${ }^{1}$ Faculty of Allied Health Sciences, Burapha University, Chonburi, Thailand \\ ${ }^{2}$ Institute of Nutrition, Mahidol University, Nakhon Pathom, Thailand
}

\begin{abstract}
Human immunodeficiency virus (HIV) infection largely impacts on the nutritional status of infected patients. At present, advance in medicine and pharmaceuticals such as antiretroviral drugs help improve health status among people living with HIV (PLHIV). Improving appetite for PLHIV by regaining their normal taste sensation and enhanced naso-oral stimulation can help promote their consumption of larger amounts of food. Previous studies have shown how bad HIV infection affects mental status, which leads to the development of many health problems including eating disorder. This is a cross-sectional study aimed to investigate characteristics of food consumed by PLHIV and comparing that between high-risk and low-risk eating disorder groups. The developed self-respondent questionnaires (baseline questionnaire, eating disorder screening questionnaire, self-adherence questionnaire, and 3-day food record) were completed by 132 HIV infected patients at the Queen Savang Vadhana Memorial Hospital, Chonburi province. The results in a high-risk group were not significant on energy distribution (carbohydrate, protein, fat) compared with a low-risk group, but the data indicated that the high-risk group had a significantly higher intake of saturated fat and sodium compared with the low-risk group. In addition, results indicated that the high-risk group was significantly lower in self-adherent score and CD4 level compared with the low-risk group.
\end{abstract}

HIV AIDS Rev 2017; 16, 2: 118-123

DOI: https://doi.org/10.5114/hivar.2017.66800

Key words: nutrition, eating disorder, self-adherence, food consumption, psychological problem.

\section{Introduction}

Human immunodeficiency virus (HIV) infection is known to be associated with impaired nutritional status that can lead to the development of acquired immune deficiency syndrome (AIDS). Loss of appetite due to loss of sensation, weight loss, and cachexia are examples of nutritional status affected by HIV infection leading to a lower quality of life, increase in opportunistic infections, and development of complications [1]. People living with HIV (PLHIV) can improve their appetite with antiretroviral drug therapy (ART) to maintain their CD4 cells and viral load at an optimal level to prevent complications from opportunistic infections (i.e., tuberculosis, pneumonia, etc.) and to keep their physiological functioning normal, especially, sensory sensations to improve taste and smell to increase their appetite and amount of food intake [2]. In Thailand, the number of PLHIV having access to ART has increased compared to the past decade due to the government universal health care program. Hence, decreased complications and nutritional
Address for correspondence: Prof. Alongkote Singhato, Faculty of Allied Health Sciences, Burapha University, 169, Long hard Bangsaen Rd., Sanssook, Mueng, 20131 Chonburi, Thailand, phone: 66851797743, e-mail: alongkote@buu.ac.th
Article history:

Received: 17.08.2016

Received in revised form: 04.01.2017

Accepted: 04.01.2017

Available online: 24.03 .2017
International Journal of HIV-Related Problem

HIV \& AIDS

R e vi e w 
status (undernutrition) of HIV infection were observed [3]. However, lipodystrophy, lipoatrophy, gastrointestinal system problems are examples of side effects commonly found in ART [4]; therefore, PLHIV need to be closely supervised and monitored by health care professionals to evaluate and assess nutritional status in order to prevent development of further complications.

Eating disorder (ED), a serious psychological problem interfering with an attitude toward food habits and self-conceived perception of weight, and body size, is commonly found in teenagers and fashion models [5]. A person who has ED is constantly concerned about his body shape and exhibits anxiety about being overweight, even at thin or normal weight. This abnormal psychological problem leads people with ED (e.g. anorexia nervosa, bulimia nervosa, etc.) to try to lose body weight by dieting, fasting, heavily exercising, using slimming pills, diuretics, laxatives, vomiting, or binge eating (consumption of an unusually large amount of food accompanied by a sense of loss of control), which can lead to development of nutritional problems such as cachexia, growth retardation, gastrointestinal and endocrine system complications [6-8]. In addition, a study revealed that HIV infection affects mental and psychological status, which can lead to further mental disorder development [9]. Screening for ED can be conducted by using validated questionnaires to investigate signs of developing $\mathrm{ED}$ such as attitude toward food consumption, changes in food preferences (e.g. refusing to eat, replacing meals with fluids, etc.), actual body weight satisfaction or weight fluctuations, and excessive exercise [10].

Monitoring nutritional status of PLHIV is essential for preventing malnutrition and keeping CD4 level and immune system supportive to the fight against complications [11]. Fortunately, advances in medical and pharmaceutical sciences help PLHIV. Most people who receive ART experience minimal drug side effects and the drugs help them have normal physiological functions, especially, sensory functions that improve taste and smell to increase appetite and enjoy food like non-infected people [12]. However, there are few studies on PLHIV with or without risk of ED and its effect on food habits and diet quality. This study is aimed to investigate characteristics of foods consumed by PLHIV with a high risk of ED by examining the relationship of ED and dietary intake.

\section{Material and methods}

This study was a cross-sectional study aimed to investigate quality of foods consumption and development of ED among PLHIV by determining the characteristics of foods being consumed using a 3-day food record which was done by using the developed self-administered questionnaire after completing the ED screening questionnaire and the HIV self-adherence questionnaire. Once completed, nutrients, energy, and self-adherence scores were analyzed. The steps in developing questionnaires and study procedures were as follows.

\section{Development of questionnaires}

\section{Eating disorder screening questionnaire}

This was a close-ended questionnaire adapted from SCOFF questionnaire [13], a validated questionnaire for screening ED. The questionnaire was carefully adapted by being translated from English to Thai language by an English native speaker who was also an expert in Thai language based at the Burapha University International College. After that, the translated questionnaire was reviewed and proofread by resident doctors in psychiatry at the Child and Adolescent Mental Health Rajanagarindra Institute. Once completed, twelve PLHIV with comparable and similar demographical characteristics and socio-economic background as the intervention participants were asked in a pretest to describe their understanding of the edited SCOFF questionnaire for revisions and feedbacks. For study methods, participants were asked to complete 5 questions about their food habits by answering "Yes" or "No". Participants who answered "Yes" to at least 2 questions were defined as at risk of ED (high risk in developing eating disorder but not yet diagnosed as such), participants who answered "Yes" to 0 or 1 question were defined as at low risk of ED (low risk in developing eating disorder but not yet diagnosed as such). Example questions include "Do you make yourself sick because you feel uncomfortably full?" and "Do you worry you have lost control over how much you eat?".

\section{HIV self-adherence questionnaire}

This was a validated visual analog scale (VAS) questionnaire asking patients about their self-adherence to drugs being administered (complete dosage, administered on time and not skipping) [14]. Participants were asked to complete this question by marking on a $100 \mathrm{~mm}$ straight line the level of their self-adherence to the taking of ART. The line starts from " 0 " for the lowest self-adherence (lowest discipline) to " 100 " for the highest self-adherence (highest discipline) (Fig. 1).

$\begin{array}{lc}\text { Lowest discipline } & \text { Highest discipline } \\ \text { ART administering } & \text { ART administering }\end{array}$

Figure 1. HIV self-adherence questionnaire scale

\section{Three-day food record}

This was an open-ended form to record participants' food consumptions for three days. Participants were trained on the food exchange list and food portion size to record their foods consumption in 2 working days and 1 weekend day [15]. Total energy, energy distribution, and nutrients were calculated using an analysis software package (INMUCALNutrient V3-version 3.0). All questionnaires were reviewed 
Table 1. Baseline data of age, body mass index, duration of receiving antiretroviral drug therapy (ART), CD4 level and viral load

\begin{tabular}{|c|c|c|c|}
\hline Variables & High risk $(n=16)$ & Low risk $(n=116)$ & Overall $(n=132)$ \\
\hline \multicolumn{4}{|l|}{ Sex } \\
\hline Male (\%) & $9(6.8)$ & $50(37.9)$ & $59(44.7)$ \\
\hline Female (\%) & $7(5.3)$ & $66(50)$ & $73(55.3)$ \\
\hline Average age, yrs (SD) & $37.2(8.3)$ & $41.7(10.0)$ & $41.1(9.9)$ \\
\hline Body mass index & $21.8(4.5)$ & $22.3(5.9)$ & $22.1(5.8)$ \\
\hline \multicolumn{4}{|l|}{ Duration of receiving ART } \\
\hline For at least 6 months (\%) & $3(2.3)$ & $16(12.1)$ & $19(14.4)$ \\
\hline $1-5$ years $(\%)$ & $5(3.8)$ & $23(17.4)$ & $28(21.2)$ \\
\hline $5-10$ years $(\%)$ & $6(4.5)$ & $43(32.6)$ & $49(37.1)$ \\
\hline More than 10 years (\%) & $2(1.5)$ & $34(25.8)$ & $36(27.3)$ \\
\hline CD4 level* (SD) & $506.7(239.6)$ & $663.6(244.0)$ & $608.2(2866.6)$ \\
\hline \multicolumn{4}{|l|}{ CDC category } \\
\hline A1 - Asymptomatic with $\geq 500$ cells/ $\mu \mathrm{l}(\%)$ & $13(9.8)$ & $106(80.3)$ & $119(90.1)$ \\
\hline A2 - Asymptomatic with $200-499$ cells/ $\mu$ l (\%) & $3(2.3)$ & $10(7.6)$ & $13(9.9)$ \\
\hline Viral load ${ }^{* *}(S D)$ & $526.4(186.2)$ & $508.1(195.0)$ & $513.4(188.3)$ \\
\hline
\end{tabular}

and proofread by experts in HIV nutrition and resident doctors in psychiatry. Once completed, the edited questionnaires were revised according to the feedbacks for use in pretest among 12 PLHIV with comparable and similar demographical characteristics and socio-economic background as the intervention participants. All questionnaires and study protocol were reviewed and approved by the ethical committee at the Queen Savang Vadhana Memorial hospital.

\section{Population}

PLHIV from the Queen Savang Vadhana Memorial hospital, Chonburi, Thailand, were recruited to the study with the following inclusion criteria: age of 18 years old and over, having received ART for at least 6 months, no oral problems that impact food intake, able to read and write Thai. Exclusion criteria include incomplete answers to questionnaires, exhibiting behavioral problems (i.e., attention deficit, hyperactive disorder [ADHD]), and failure to follow up the participants.

\section{Data collection and procedures}

Convenient samplings were conducted to select participants. PLHIV who came to receive health service at the Outpatient Department (OPD) of the Queen Savang Vadhana Memorial Hospital were invited to participate in this study and were informed of the criteria. Participants received an introductory letter and completed informed consent before being included in the study. Participants were trained on food exchange list and food portion size by professionals in nutrition and dietetics. Once completed, all selected participants took two consecutive days to complete the study.

\section{Baseline data collection and eating disorder screening}

At baseline, all selected participants were invited to a room at the Queen Savang Vadhana Memorial Hospital to complete the baseline data questionnaires (including age, gender, etc.) and ED screening questionnaire to screen the risk of ED. Once completed, all selected participants were assigned to record their food consumption for 3 days ( 2 working days and 1 weekend day). In addition, participants were also asked to complete the self-adherence questionnaire to investigate their discipline of ART drug administering.

\section{Data collection on food quality consumed}

One week after baseline, participants were re-invited to the Queen Savang Vadhana Memorial Hospital to submit their 3-day food record. Food consumption data were collected to calculate total energy, energy distribution, and nutrients using a nutritional software package.

\section{Statistical analysis}

Mean scores $( \pm$ SD) of age, body mass index, CD4 level, self-adherence scores, total energy, energy distribution, and nutrients were determined. Independent paired $t$-test was used to compare the test mean scores between high-risk and low-risk ED groups. Statistical analyses were performed by using the predictive analytics software statistics (PASW) 
Table 2. Food quality consumed by participants and their CD4 level and viral load

\begin{tabular}{|c|c|c|c|}
\hline Variables & High risk & Low risk & $P$ value* \\
\hline Body mass index (SD) & $21.8(4.5)$ & $22.3(5.9)$ & 0.76 \\
\hline Average age, in years (SD) & $37.2(8.3)$ & $41.7(10.0)$ & 0.04 \\
\hline \multicolumn{4}{|l|}{ Energy and nutrient consumed } \\
\hline Energy, kcal (SD) & $1,463.4(198.4)$ & $1,369.0(203.4)$ & 0.84 \\
\hline Energy from fat, \% (SD) & $30.8(3.8)$ & $29.7(4.2)$ & 0.43 \\
\hline Energy from carbohydrate, \% (SD) & $51.9(6.9)$ & $53.0(6.5)$ & 0.36 \\
\hline Energy from protein, \% (SD) & $17.3(4.1)$ & $17.4(4.8)$ & 0.11 \\
\hline Energy from saturated fat, \% (SD) & $18.0(3.5)$ & $11.8(4.6)$ & $<0.00$ \\
\hline Daily sodium intake, mg (SD) & $3,408.5(252.3)$ & $3,042.7(275.5)$ & $<0.00$ \\
\hline Self-adherence ${ }^{\star \star}, \mathrm{mm}(\mathrm{SD})$ & $81.3(5.2)$ & $90.1(5.9)$ & 0.04 \\
\hline CD4 level, cells/mm³ (SD) & $506.7(239.6)$ & $663.6(244.0)$ & 0.02 \\
\hline Lowest CD4 T cell count, cells/ml & $334(98.2)$ & $364(102.5)$ & 0.86 \\
\hline Viral load, copies/ml (SD) & $526.4(186.2)$ & $508.1(195.0)$ & 0.72 \\
\hline
\end{tabular}

dependent paired t-test

${ }^{* *}$ Data from $100 \mathrm{~mm}$ straight line

version 21.0 (SPSS Inc., Chicago, IL, USA). Statistical significance was established at $p<0.05$.

\section{Results}

There were 132 PLHIV recruited. At baseline, twelve percent of selected participants $(n=16)$ were defined as the high-risk ED group. Most of them (55.3\%) were female with an average age of 41 years. The average body mass index (22.1) indicated that most participants were classified as having normal weight and most had received ART for 5-10 years (37.1\%). Most of participants (90.1\%) were in A1 (Asymptomatic with $\geq 500$ cells $/ \mu \mathrm{l}$ ), and their averages CD4 level and viral load were 608 cells/mm3 and 513 copies $/ \mathrm{ml}$, respectively (Table 1 ).

For food intake, results revealed no significant differences between the high-risk ED group and the low-risk ED group for body mass index, total energy intake, energy distribution on fat, carbohydrate, protein, lowest CD4 T cell count, and viral load. These indicated the risk of ED development did not impact body mass index, total energy intake, energy distribution on fat, carbohydrate, protein, and viral load among PLHIV; however, results revealed the high-risk ED group had significantly higher intake of saturated fat (18.0 \pm 3.5 vs. $11.8 \pm 4.6, p \leq 0.00)$ and sodium $(3,408.5 \pm 252.3$ vs. $3,042.7$ $\pm 275.5, p \leq 0.00)$ compared with the low-risk ED group, which indicated PLHIV with high risk of ED development were considered to be consuming poorer quality food with high saturated fat and sodium. In addition, results revealed that the high-risk ED group had significantly lower age (37.2 \pm 8.3 vs. $41.7 \pm 10.0, p=0.04)$ and CD4 level $(506.7 \pm 239.6$ vs. $663.6 \pm 244.0, p=0.02)$ compared with the low-risk ED group which increased the risk of developing further complications. Moreover, results indicated PLHIV in the low- risk ED group had significantly higher self-adherence scores $(81.3 \pm 5.2$ vs. $90.1 \pm 5.9, p=0.04)$ compared with the highrisk ED group (Table 2).

\section{Discussion}

This study aimed to investigate characteristics of food intake of PLHIV in high-risk ED and low-risk ED groups by determining and comparing their food energy and nutrient consumption. Results indicated 16 participants were defined as high risk of ED development and most of them were male. This finding was in contrast with a previous study that revealed females have a higher risk of developing ED (energy restriction) than males [16]. However, our results were still preliminary for risk of ED development and participants in the high-risk ED group need further diagnosis by psychiatry experts to confirm their ED status. Gladly, most of participants were in A1 according to CDC category which indicated that PLHIV in this area had good and accessible health care services as well as a lower risk of complications, such as dermatological disorders that was reported as one of the major health problems among PLHIV in Thailand [17]. Result showed significantly lower age in the high-risk ED group compared with the low-risk ED group, which agrees with the finding of a previous study that a high risk of ED was prevalent in young people. Therefore, young PLHIV need to be closely supervised by professional health care providers and their family members for nutritional counseling and mental support [18].

For food quality consumption, results revealed no significant difference in total energy intake and body mass index between the groups. This result indicated that the risk of ED did not impact undernutrition and nutrient deficiency development. In contrast, a previous study revealed peo- 
ple with ED usually limited their food and energy intake, which can lead to nutrient deficiency and cachexia development [19]. The findings of this study showed a significantly higher amount of saturated fat and sodium consumption in the high-risk ED group compared with the low-risk ED group, which indicated that PLHIV with a higher risk of ED have a higher risk of developing non-communicable diseases than those with a low risk of ED. Previous studies revealed that long-term high intake of saturated fat can lead to dyslipidemia and cardiovascular diseases and longterm high intake of sodium can lead to hypertension and chronic kidney disease [20, 21]. Thus, PLHIV with a high risk of ED development have a high risk of complications from non-communicable diseases, which can increase their mortality rate. The increased mortality from non-communicable diseases in people with ED was not only restricted to HIV infected patients. A previous study revealed patients with other chronic diseases, such as type 2 diabetes, with a risk of ED development had also an increased mortality rate due to a significantly higher intake of fat and saturated fat compared with type 2 diabetes patients with a low risk of ED development [22]. In addition, our results indicated the average daily sodium consumption was 3,000 mg per day in both high-risk ED and low-risk ED groups, which is similar to the findings of previous studies on intake of sodium in Thailand that is limited to $2,400 \mathrm{mg}$ per day according to the guideline $[23,24]$.

In addition, the findings of this study indicated no significant difference in viral load copies between the two groups. However, our results revealed that PLHIV with a low risk of ED had significantly higher self-adherence scores compared to those with a high risk of ED, corresponding with the high discipline of ART administering among PLHIV with a low risk of ED. The CD4 level was also significantly higher in the high-risk ED group, which may help prevent opportunistic infections [25]. The limitations of this study were that information on participants' physical activity was not collected and just one food record was collected for each participant, which might not be enough to validate the composition of food being consumed because they may consume different food during other working days and weekend days. Therefore, further studies are needed to identify other factors that could affect body weight and energy expenditure, and there is also a need to collect more food records for each participant.

\section{Conclusions}

This study investigated food quality consumed by PLHIV with a risk of ED by examining the relationship of ED and dietary intake. The findings revealed that PLHIV with a high risk of ED consumed significantly more saturated fat and sodium compared to those with a low risk of ED, which indicated that PLHIV had a higher risk of non-communicable diseases caused by a high intake of saturated fat and sodium which lead to an increase in mortality. The results also revealed PLHIV in the high-risk ED group had significantly lower self-adherence scores and discipline in ART administering compared with PLHIV in the low-risk ED group which is related to their significantly lower CD4 level. Therefore, PLHIV with ED risk had a higher risk of complications from opportunistic infections compared with PLHIV with a low risk of ED. This study showed an increased health risk of PLHIV with a high risk of ED, which resulted in a higher mortality rate; hence, caregivers and professionals in healthcare services need to closely monitor PLHIV food habits and stay alert on their ED development and their self-adherence to drug administration. Also, both groups of PLHIV need to receive nutritional counseling to improve the quality of food being consumed, particularly to avoid a high amount of sodium intake.

\section{Conflict of interest}

The authors declared no potential conflicts of interest with respect to the research, authorship, and/or publication of this article.

\section{References}

1. Obi SN, Ifebunandu NA, Onyebuchi AK. Nutritional status of HIVpositive individuals on free HAART treatment in a developing nation. J Infec Develop Countries 2010; 4: 745-749.

2. Martinez H, Palar K, Linnemayr S, et al. Tailored nutrition education and food assistance improve adherence to HIV antiretroviral therapy: evidence from Honduras. AIDS Behav 2014; 18: 566-577.

3. The Joint United Nations Programme on HIV/AIDS (UNAIDS). Thailand AIDS response progress report 2015. Reporting Period: Fiscal Year of 2014.

4. Lana LG, Junqueira DR, Perini E, et al. Lipodystrophy among patients with HIV infection on antiretroviral therapy: a systematic review protocol. Br Med J Open 2014; 4: e004088.

5. Smith H. Anorexia Nervosa and Eating Disorder. South African Pharmaceutical Journal 2012; 79: 34-36.

6. Keel PK, Klump KL. Are eating disorders culture-bound syndromes?: Implications for conceptualizing their etiology. Psychol Bull 2003; 129: 747-769.

7. Katzman DK. Medical Complications in Adolescents with Anorexia Nervosa: A Review of the Literature. Int J Eating Dis 2005; 37 : 52-59.

8. Mitchell JE, Crow S. Medical complications of anorexia nervosa and bulimia nervosa. Curr Opin Psychiatry 2006; 19: 438-443.

9. Chandra PS, Desai G, Ranjan S. HIV \& psychiatric disorder. Indian J Med Res 2005; 121: 451-467.

10. Yeo M, Hughes E. Eating disorders: early identification in general practice. Australian Family Physician 2011; 40: 108-111.

11. Paton N, Sangeetha S, Earnest A, et al. The impact of malnutrition on survival and the CD4 count response in HIV-infected patients starting antiretroviral therapy. HIV Med 2006; 7: 323-330.

12. Rehman AM, Woodd S, Chisenga M, et al. Appetite testing in HIVinfected African adults recovering from malnutrition and given antiretroviral therapy. Public Health Nutrition 2015; 18: 742-751.

13. Morgan JF, Reid F, Lacey JH. The SCOFF questionnaire: a new screening tool for eating disorders. West J Med 2000; 172: 164-165.

14. Johnson MO, Neilands TB, Dilworth S, et al. The Role of Self-Efficacy in HIV Treatment Adherence: Validationof the HIV Treatment Adherence Self-Efficacy Scale (HIV-ASES). J Behav Med 2007; 30: 359-370.

15. Sammasud R. Thai Food Exchange List. Thai Journal of Parenteral and Enteral Nutrition 2004; 15. 
16. Striegel-Moore RH, Rosselli F, Perrin N, et al. Gender Difference in the Prevalence of Eating Disorder Symptoms. Int J Eating Dis 2009; 42: 471-474.

17. Wiwanitkit V. Prevalence of dermatological disorders in Thai HIVinfected patients correlated with different CD4 lymphocyte count statuses: a note on 120 cases. Int J Dermatol 2004; 43: 265-268.

18. Gowers S, Bryant-Waugh R. Management of child and adolescent eating disorders: the current evidence base and future directions. J Child Psychol Psychiatry 2004; 45: 63-83.

19. Racz O, Kuzmova D, Fodor B. Nutrient requirements, undernutrition and eating disorders. Egészségtudományi Közlemények (Health Publication Journal) 2012; 2: 63-68.

20. O'Sullivan TA, Hafekost K, Mitrou F, et al. Food sources of saturated fat and the association with mortality: a meta-analysis. Am J Public Health 2013; 103: 31-42.

21. He FJ, MacGregor GA. A comprehensive review on salt and health and current experience of worldwide salt reduction programmes. J Hum Hypertens 2009; 23: 363-384.

22. Tse J, Nansel TR, Haynie DL, et al. Disordered eating behaviors are associated with poorer diet quality in adolescents with type 1 diabetes. Journal of the Academy of Nutrition and Dietetics 2012; 112: 1810-1814.

23. The Thai Hypertension Society. Thai Guidelines on the Treatment of Hypertension Update 2015. Trickthink Printing, Chiangmai 2015.

24. Buranakitjaroen P, Phoojaroenchanachai M. The prevalence of high sodium intake among hypertensive patients at hypertension clinic, Siriraj Hospital. Journal of the Medical Association of Thailand 2013; 2: 1-8.

25. Patrikar S, Basannar DR, Bhatti VK, et al. Rate of decline in CD4 count in HIV patients not on antiretroviral therapy. Armed Forces Med J India 2014; 70: 134-138. 